\title{
A Case Report: Aggressive Pyogenic Granuloma
}

\author{
Dipanshu Kumar ${ }^{1}$ Tanya Agarwal ${ }^{2}$ \\ ${ }^{1}$ Department of Pedodontics \& Preventive Dentistry, Inderprastha \\ Dental College and Hospital, Ghaziabad, Uttar Pradesh, India \\ 2Department of Pedodontics \& Preventive Dentistry, Institute of Dental \\ Studies and Technologies, Kadrabad, Modinagar, Uttar Pradesh, India
}

\begin{abstract}
Address for correspondence Tanya Agarwal, Department of pedodontics \& preventive dentistry, Institute of Dental Studies and Technologies, Kadrabad, Modinagar, Uttar Pradesh 201204, India (e-mail: tanyapedo@gmail.com).
\end{abstract}

Dent J Adv Stud 2019;7:46-50

\begin{abstract}
Keywords

- pyogenic granuloma

- oral cavity

- posterior teeth

- reactive hyperplasia

Soft-tissue growths of the oral cavity offer a diagnostic dilemma because of the pathologic processes produced by such lesions. Pyogenic granuloma (PG) is one such soft-tissue swelling that originates from the reactive inflammatory course in which an exuberant fibrovascular expansile growth of the connective tissue of the skin or mucous membrane occurs. This case report documents a rare case in which the size and the site of the PG were different from the usual. The treatment included the extraction of the involved tooth in addition with the surgical removal of the lesion. The present case report demonstrates that the treatment of choice varies according to individual case after the adequate analysis of clinical and radiographic findings and should be managed appropriately to minimize further reoccurrence of the lesion and postsurgical complications.
\end{abstract}

\section{Introduction}

Pyogenic granuloma (PG) is a common non-neoplastic inflammatory hyperplastic enlargement seen inside the oral cavity as a tissue reaction to irritation, trauma, or hormonal imbalances and was first elaborated by Hullihen in 1844. ${ }^{1,2}$ It emanates as an elevated sessile or pedunculated growth, surfaced with red hemorrhagic and erythematous papules covered by a fibrinous membrane that usually shows ulcerations. The color divaricates from purple pink to red, based on the vascularity of the lesion. The most common site in the oral cavity is gingiva, usually the marginal gingiva, and is seen majorly in the maxillary gingiva. Moreover, the anterior areas are commonly affected than posterior areas of the oral cavity. Clinically, the lesion rarely exhibits rapid growth, is symptomless, and does not cause pain. ${ }^{3}$ The radiographic findings are typically absent, but in long standing gingival PGs, localized alveolar bone resorption can be seen. ${ }^{4}$

Biopsy findings have an important role and are definitive in establishing the diagnosis. The treatment involves excision of the lesion along with histopathological evaluation to confirm the diagnosis. One important aspect during the excision of PG is careful monitoring during surgical removal because of its vascular nature leading to profuse bleeding. ${ }^{5}$ The present case documents a rare case of aggressive PG associated with the gingiva of mandibular permanent first and second molar in an 11-year-old girl.

received

August 24, 2018

accepted after revision

February 27, 2019

\section{Case Report}

An 11-year-old female patient reported to the outpatient Department of Pedodontics with the chief complaint of growth in the oral cavity pertaining to lower right back tooth region which bled often and interfered with normal eating and brushing. She noticed the growth 20 days back which started as a size of peanut and gradually increased to reach the present size. The medical and family history were non-contributory. On extraoral examination, swelling was seen on the lower right side of the face in relation to the inferior border of the mandible extending posteriorly. The intraoral examination revealed a single growing exophytic lesion measuring approximately 2 by $2 \mathrm{~cm}$ which was attached to the marginal gingiva extending between the right mandibular first molar and second molar (-Fig. 1). The lesion was reddish in color, oval in shape, pedunculated with a smooth surface, and bleeding was present on probing. There was noticeable mobility of the mandibular first molar, and these findings were confirmed by palpation of the lesion. Correlating the history and intra-oral findings, a provisional diagnosis of pyogenic granuloma was made.

Intraoral periapical radiograph of teeth no. 46 and no. 47 and orthopantomogram revealed interproximal alveolar crestal bone loss and root resorption associated with no. 46 ( - Fig. 2A and B). After explaining the treatment plan to the parents and obtaining the consent, oral 
prophylaxis was done followed by the extraction of no. 46 along with the surgical excision of the lesion ( - Fig. $2 \mathrm{C}$ ). The postoperative instructions were given and the excised tissue specimen was sent for histopathological assessment. The histological section at different magnification (10X and 40X) showed hyperplastic parakeratinized stratified

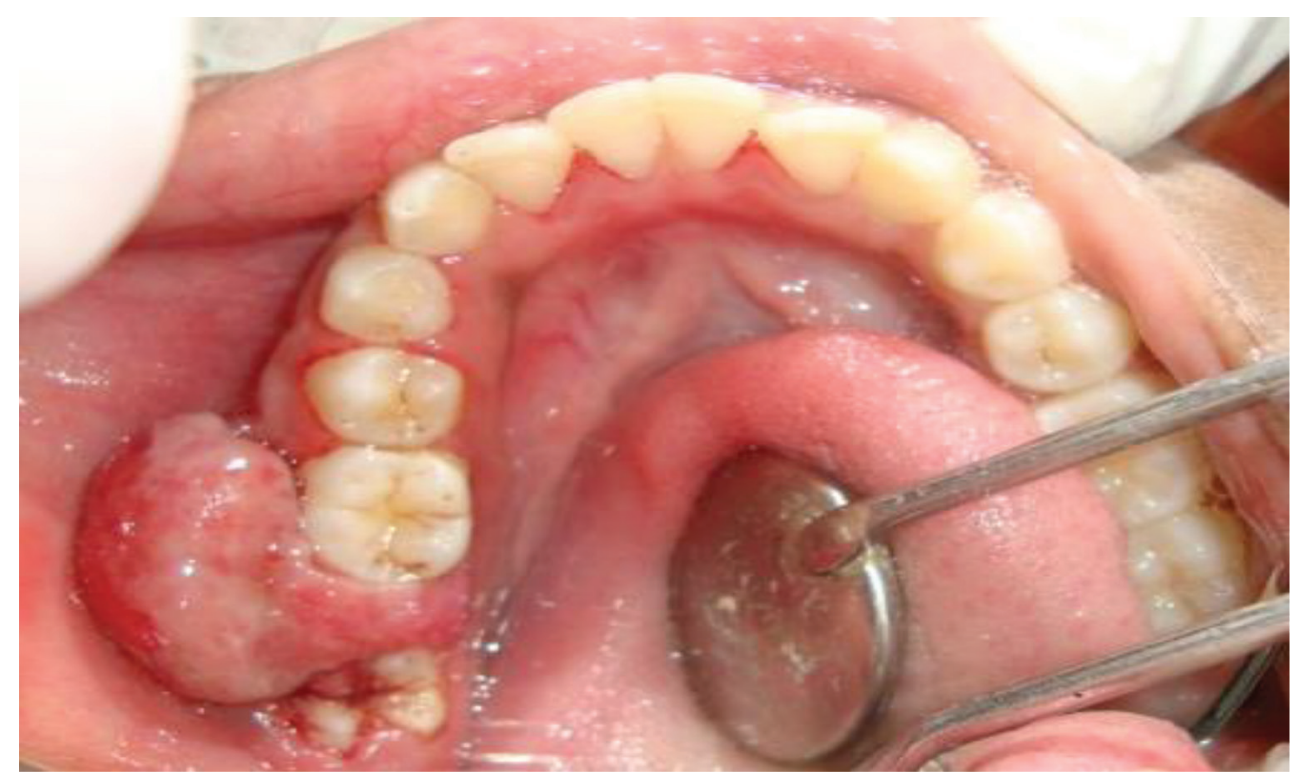

Fig. 1 Preoperative view showing pyogenic granuloma attached to the marginal gingiva extending between the right mandibular first molar and second molar.
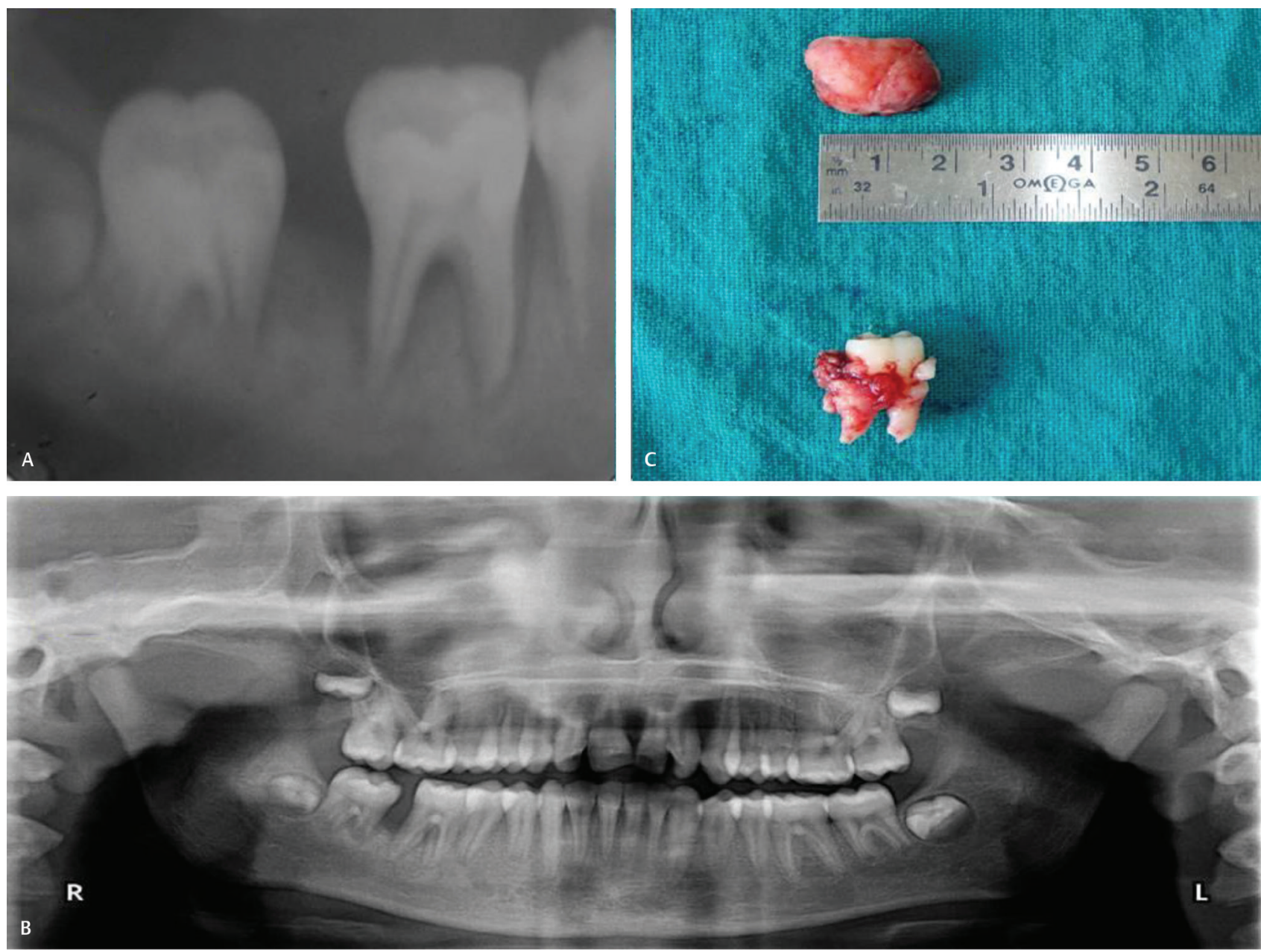

Fig. 2 (A, B) IOPA and OPG showing root resorption of right mandibular first molar and interproximal alveolar crestal bone loss. (C) Excised lesion. 
squamous epithelium with supporting fibrovascular connective tissue consisting of many budding capillaries, plump fibroblasts, extravasated red blood cells and dense chronic inflammatory cell infiltrate ( - Fig. 3A and B). Thus, a final diagnosis of pyogenic granuloma was given. After 1 week, the patient was reviewed and an uneventful and satisfactory postoperative healing was noticed (-Fig. 4).

\section{Discussion}

PG is a type of inflammatory hyperplasia with various terminologies such as granuloma pyogenicum, eruptive hemangioma, and granuloma gravidarum. ${ }^{6}$ Moreover, PG is a misnomer since the lesion does not consist of any pus and histologically also is not a granuloma. ${ }^{7}$
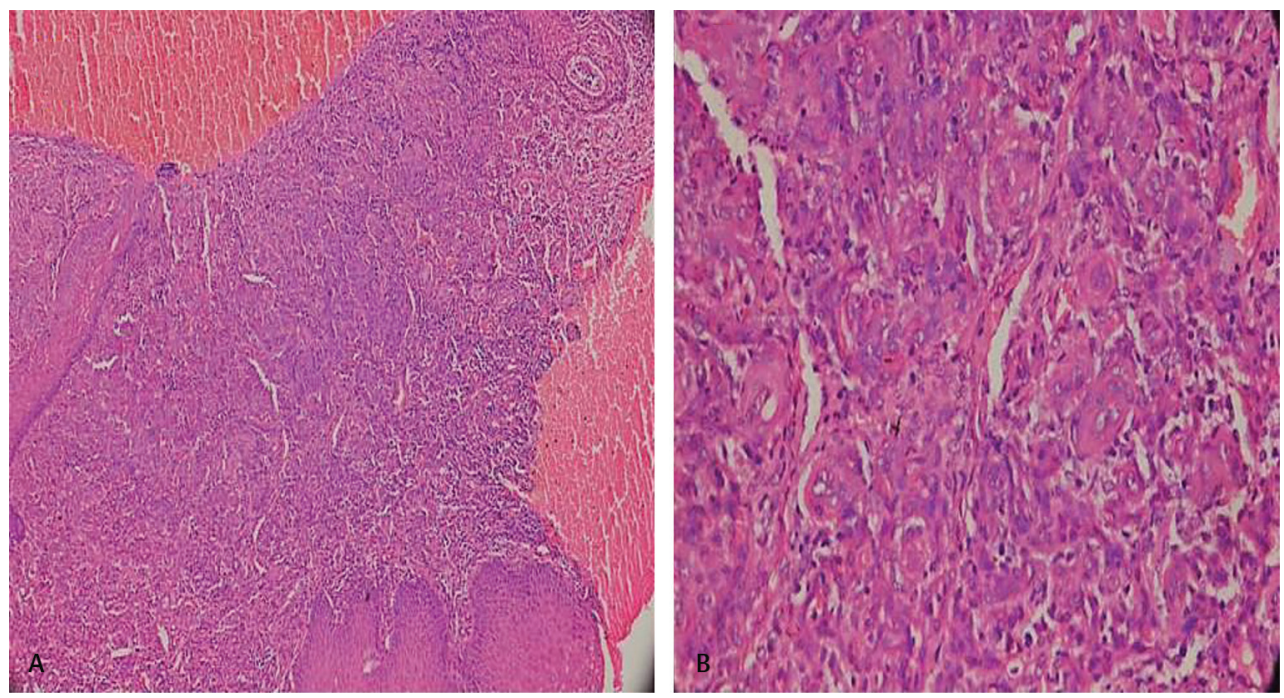

Fig. 3 Histological section of the lesion at (A) 10X (B) 40X. At 10x: low power view showing overlying ulcerated stratified squamous epithelium with areas of high proliferation. Supporting connective tissue stroma demonstrates highly proliferative vascular tissue. Budding vascular channels are arranged in lobular pattern. Evidence of inflammatory reaction with numerous inflammatory cell infiltration around the capillaries and throughout the stroma can be seen. At 40x: high power view showing numerous budding capillaries engorged with red blood cells. These capillaries are lined by plump endothelial cells. Proliferating endothelial cells are evident. These are surrounded by mixed inflammatory cell infiltrate showing neutrophils, plasma cells and lymphocytes.

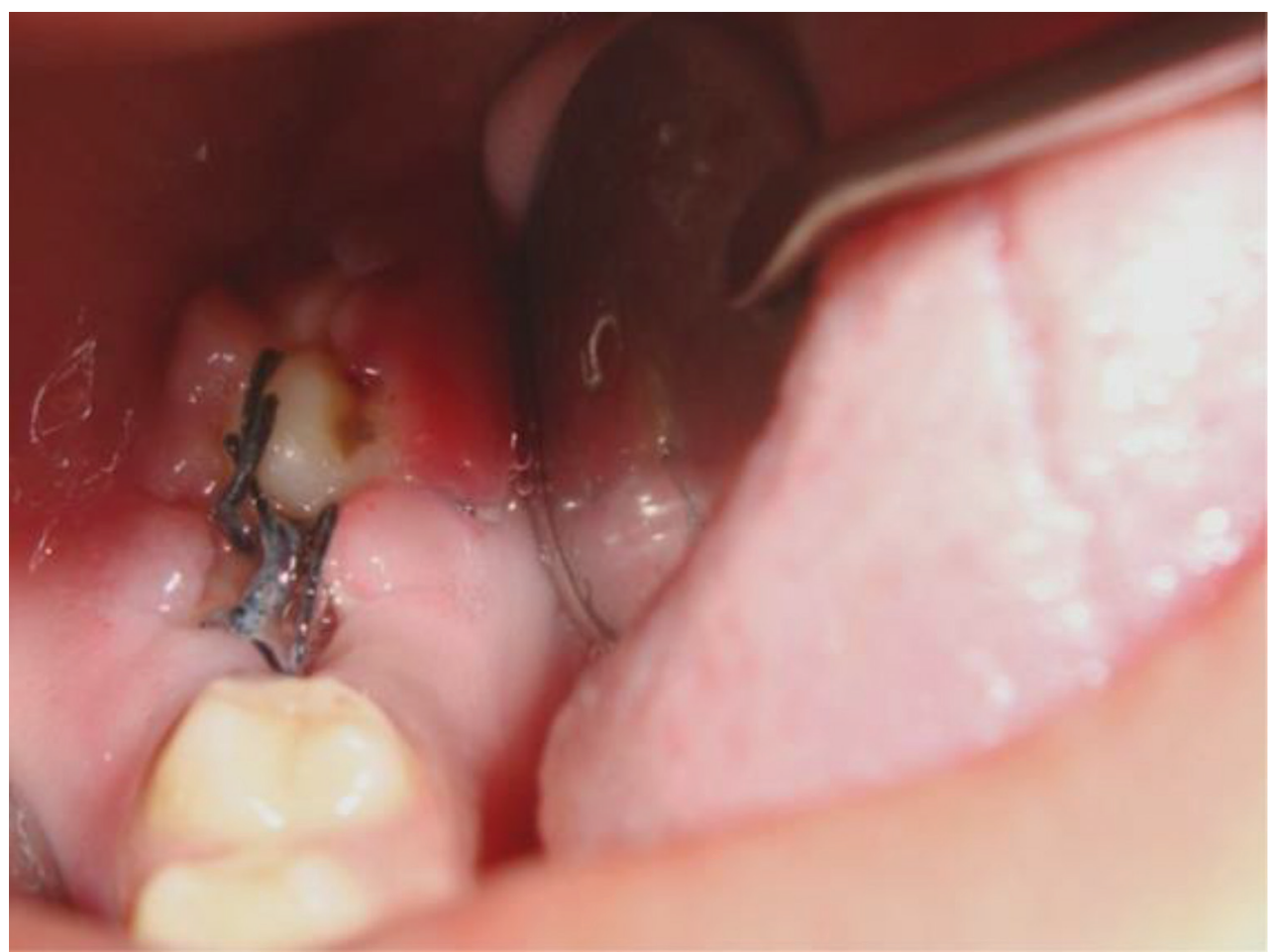

Fig. 4 Postoperative view showing uneventful healing after 1 week. 
Pyogenic granuloma is a commonly occurring reactive lesion of the oral cavity. It is non-neoplastic in nature and is hence referred to as a tumor-like lesion. Histologically, it was described by Angelopoulos as hemangiomatous granuloma because of its inflammatory nature and presence of multitudinal blood vessels. ${ }^{8,9}$ Histopathologically, pyogenic granuloma is classified into lobular capillary hemangioma (LCH) and nonlobular capillary hemangioma (non-LCH). Proliferating blood vessels in lobular aggregates, with no specific changes such as edema and capillary dilatation is seen in LCH whereas in Non-LCH type a vascular core resembling granulation tissue with foci of fibrous tissue is seen with greater number of blood vessels. Oral PGs are mainly of LCH type. The microscopic picture of pyogenic granuloma in general shows exuberant granulation tissue which is covered by atrophic/hyperplastic epithelium that may be ulcerated at times and reveals fibrinous exudates. Presence of numerous endothelium-lined vascular spaces and proliferation of fibroblasts and budding endothelial cells are the characteristic features of pyogenic granuloma. Presence of mixed inflammatory cell infiltration is also observed.

At present it is now acknowledged that pyogenic granuloma occurs as a consequence of different stimuli such as hormonal imbalances, low grade chronic irritation, trauma or certain type of drugs and oral contraceptives. Nonspecific bacterial infection is supposed to be of minor involvement rather than being the main etiology of this lesion. The tissues respond in a typical process ensuing in excessive and uncontrolled growth of the vascular content of connective tissue. ${ }^{10}$ Oral PGs can appear in all age groups from children to adults, but commonly seen in females in the second decade of life due to the rise in levels of hormones. Pyogenic granuloma generally affects the gingiva primarily, especially the marginal gingiva and is more common in maxillary than mandibular gingiva. Anterior areas are more frequently affected than posterior areas. All these lesions are more common on the facial than the lingual aspect whereas in the present case report the aggressive nature and the unusual presentation of the lesion is evident from its location and size as it is seen in the mandibular posterior region extending from buccal to lingual aspect. It was also observed that the aggressive pyogenic granuloma was causing alveolar bone resorption, thus displacing teeth in the mandibular posterior region. Similar such finding was reported by Saravana in his review of 137 cases reported four patients with extensive bone loss and mobility of teeth. ${ }^{11}$

Differential diagnosis includes hemangioma, peripheral giant cell granuloma, hyperplastic gingival inflammation, pregnancy tumor, peripheral ossifying fibroma, bacillary angiomatosis, angiosarcoma, metastatic cancer, Kaposi's sarcoma, and non-Hodgkin's kymphoma. ${ }^{12}$ Peripheral giant cell granuloma is almost clinically identical to PG; however, bone resorption in radiograph and appearance of the multinucleated giant cell are differentiating features. ${ }^{3}$ Also, fibroma can be distinguished by the consistency, texture, and the lighter color. Metastatic tumors, even though clinically resembles PG, the microscopic features look like as the tumor of origin. ${ }^{13}$
Depending on the extent of the lesion management varies and with increase in size of the lesion, occlusal interference during brushing and eating is seen. As a result there will be release of angiogenic and endogenous factors leading to the rise in blood supply to the affected region consequently resulting in bleeding. Levels of estrogen and progesterone hormones will be augmented during second decade of life especially in females but it can be seen in all age groups. Cryosurgery, flash lamp pulsed dye laser, sclera therapy, excision by Nd YAG laser, injection of corticosteroid or ethanol are additional treatment modalities for pyogenic granuloma. ${ }^{14}$ In the presented case the size of the lesion was moderate; therefore, surgical excision was carried out which is also a recommended treatment in the dental literature.

While treating such lesions, importance on maintenance of oral hygiene should be advised. This benign vascular neoplasm does not undergo malignant transformation, but it tends to recur occasionally after surgical excision usually as a result from incomplete excision, failure to remove etiologic factors, or reinjury of the area. It has also been noted that gingival lesions show a much higher recurrence rate than lesions from other oral mucosal sites. ${ }^{15,16}$ It was also observed that there was no reoccurrence of the lesion after 1 year of follow-up.

\section{Conclusion}

Soft-tissue enlargements such as pyogenic granuloma although non-neoplastic can be present at unusual site with atypical size, consequently, appropriate management pertaining to its diagnosis, treatment, and further prevention is extremely imperative.

\section{Authors' Contributions}

Dr. Dipanshu kumar conceived the idea, and helped in the analysis of data and final editing. Dr. Tanya Agarwal helped in collection of data, sample formation, analysis of data, and manuscript preparation.

\section{Conflict of Interest}

None declared.

\section{References}

1 Greenberg MS, Glick M. Burkett's Oral Medicine: Diagnosis and Treatment. 10stedn. Hamilton, Canada: BC Decker; 2003

2 Hullihen SP. Case of aneurism by anastomosis of the superior maxillae. Am J Dent Sci 1844;4(3):160-162

3 Regezi JA, Sciubba JJ, Jordan RC. Oral Pathology and Clinical Pathological Considerations. 4th ed. Philadelphia, PA: W B Saunders; 2003:115-6

4 Ganesan A, Kumar NG, Azariah E, Asokan GS. Oral pyogenic granuloma: a case report and a comprehensive review. SRM J Res Dent Sci. 2015;6:250-256

5 Mahajan A, Singhal P, Poonam. Lobular capillary hemangioma in a child: a case report and literature review. Open Access Scientific Reports 2012;1:265. doi:10.4172/scientificreports.265

6 Jafarzadeh H, Sanatkhani M, Mohtasham N. Oral pyogenic granuloma: a review. J Oral Sci 2006;48(4):167-175 
7 Bouquot JE, Nikai H. Lesions of oral cavity. In: Gnepp DR, ed. Diagnostic Surgical Pathology of Head and Neck. Philadelphia, PA: W B Sanders; 2001:141-233

8 Angelopoulos AP. Pyogenic granuloma of the oral cavity: statistical analysis of its clinical features. J Oral Surg 1971;29(12):840-847

9 Gomes SR, Shakir QJ, Thaker PV, Tavadia JK. Pyogenic granuloma of the gingiva: A misnomer? A case report and review of literature. J Indian Soc Periodontol 2013;17(4):514-519

10 Shafer WG, Hyne MK, Lcvy HM. Shafer's textbook of Oral Pathology. 4stedn, Philadelphia, London, Toronto: WB Saunders; 1983

11 Saravana GH. Oral pyogenic granuloma: a review of 137 cases. Br J Oral Maxillofac Surg 2009;47(4):318-319

12 Neville BW, Damm DD, Allen CM, Bouquot JE. Oral and Maxillofacial Pathology. 2nd ed. Philadelphia, PA: Saunders; 2002:437-95
13 Nirmala SVSG, Vallepu R, Babu M, Dasarraju RK. Pyogenic granuloma in an 8-year-old boy: a rare case report. J Pediatr Neonatal Care 2016;4(2):00135

14 Parajuli R, Maharjan S. Unusual presentation of oral pyogenic granulomas: a review of two cases. Clin Case Rep 2018;6(4):690-693

15 Rosa CG, Lay AC, Torre ACL. Oral pyogenic granuloma diagnosis and treatment: a series of cases. Rev Odontol Mex 2017;21(4):244-252

16 Fowler EB, Cuenin MF, Thompson SH, Kudryk VL, Billman MA. Pyogenic granuloma associated with guided tissue regeneration: a case report. J Periodontol 1996; 67(10):1011-1015 